\title{
Detection of incorrect manufacturer labelling of hip components
}

\author{
Matthieu Durand-Hill $^{1}$. Johann Henckel ${ }^{1}$ - Matthew Burwell ${ }^{2}$ John Skinner ${ }^{1}$. \\ Alister Hart ${ }^{1}$
}

Received: 7 February 2016 /Revised: 22 August 2016 / Accepted: 30 August 2016/Published online: 22 September 2016

(C) The Author(s) 2016. This article is published with open access at Springerlink.com

\begin{abstract}
We describe the case of a 53-year-old man who underwent a left metal-on-metal hip resurfacing in 2015. Component size mismatch (CSM) was suspected because of the patient's immediate post-operative mechanical symptoms and high metal ion levels. Surgical notes indicated the appropriate combinations of implants were used. However, we detected a mismatch using computed tomography. Revision was performed and subsequent measurements of explanted components confirmed the mismatch. To our knowledge, this case is the first report of a CT method being used in a patient to preoperatively identify CSM.
\end{abstract}

Keywords Computed tomography $\cdot \mathrm{X}$-ray $\cdot$ Arthroplasty $\cdot$ Replacement $\cdot$ Hip $\cdot$ Prostheses and implants $\cdot$ Postoperative care $\cdot$ Hip prosthesis

\section{Introduction}

Size mismatch of components used in total hip arthroplasty, is a "serious, largely preventable" incident that has devastating effects for patients [1]. The risk of component size mismatch

Electronic supplementary material The online version of this article (doi:10.1007/s00256-016-2478-4) contains supplementary material, which is available to authorized users

Matthieu Durand-Hill

zchahm8@ucl.ac.uk

1 Institute of Orthopaedics, University College London, London, UK

2 Royal United Hospital, Bath, UK
(CSM) is predicted to rise, owing to growing numbers of increasingly complex components being used in orthopaedics [2]. The National Joint Registry for England, Wales and Northern Ireland reported that 62 out of the 9,676 revisions performed in 2013 were due to CSM of the head-acetabular socket $(0.64 \%)$ [3]. However, it is likely that CSM is underestimated (with an incidence closer to $1 \%$ ), because of difficulties in intra-operative and plain radiograph detection [2].

The detection of metal-on-metal (MoM) CSM using plain radiographs remains challenging (Figs. 1, 2). In a recent survey, the mean detection rate, by surgeons using plain radiographs, of MoM CSM was $27.7 \%$ (see Appendix) [2]. The low contrast between the cup and head component in MoM hip replacements, combined with the low intended clearance of bearing surfaces (approximately $200 \mu \mathrm{m}$ ) explains the difficulty in radiograph detection. In contrast, detection of metalon-polyethylene CSM is possible using plain radiographs, and has been reported previously $[4,5]$.

To our knowledge, this case is the first report of CT being used in a patient to pre-operatively identify CSM. This manuscript does not address the medico-legal implications of this issue.

\section{Case report}

In 2015, a 53-year-old man (with previous right hip resurfacing) underwent a MoM hip resurfacing for left hip osteoarthritis. Intra-operatively, the consultant orthopaedic surgeon (who has 17 years' experience performing total hip resurfacing) suspected that the head was undersized compared with the acetabular cup. The component's engravings could not be visualised intra-operatively. The implant manufacturer was informed via the representative, 


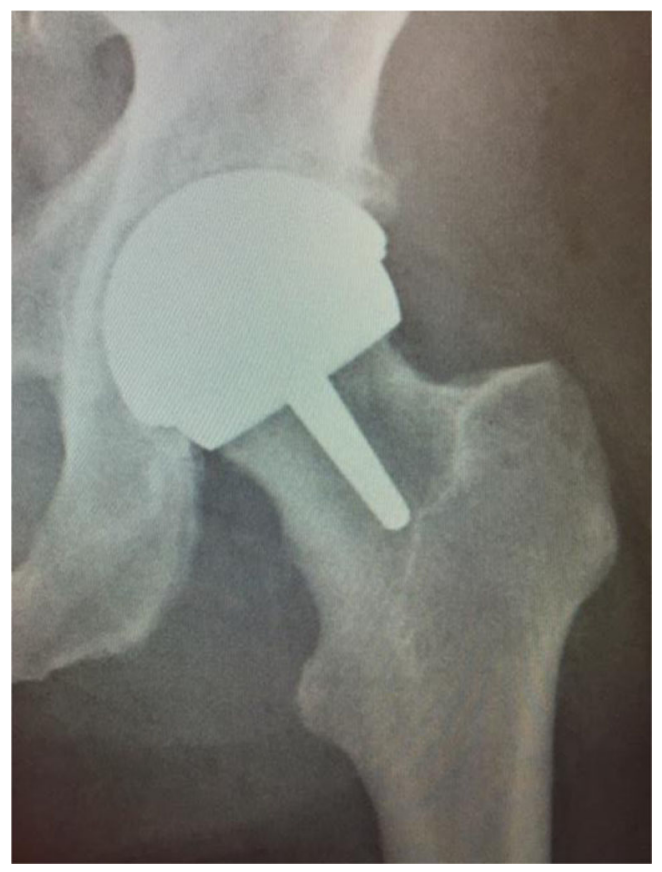

Fig. 1 Post-operative anterior-posterior plain film radiograph of the patient's left hip. No cause of the patient's mechanical symptoms was detected

but reported that the size of the components would be as marked on the boxes. Hence, the components were left in situ. The acetabular cup's outer diameter was recorded (using the manufacturer's labels) as $56 \mathrm{~mm}$, the acetabular bearing surface diameter was $48 \mathrm{~mm}$ and the femoral head component's diameter was recorded as $48 \mathrm{~mm}$.

Anterior-posterior and lateral X-rays of the patient's hip were acquired (Figs. 1, 2). No obvious abnormalities were seen on the radiographs and the patient was subsequently discharged with an early 2-week review, because of the surgeon's initial intra-operative suspicion of CSM. At 2 weeks the patient's incision had healed and the patient reported mechanical symptoms of instability and "squelching" of the component. This was very dissimilar to the immediate post-

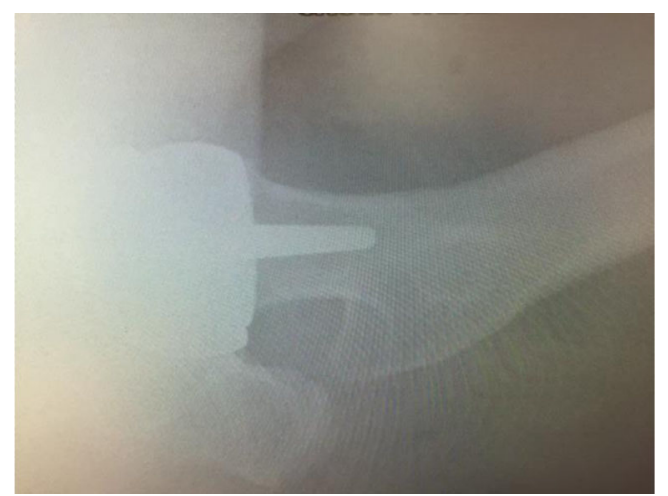

Fig. 2 Post-operative lateral plain film radiograph of the patient's left hip. No cause of the patient's mechanical symptoms was detected operative symptoms of the previous contralateral hip resurfacing.

Blood cobalt and chromium levels were measured at 5 weeks post-operatively. Five-week metal ion results were significantly elevated: cobalt was $69.3 \mathrm{ppb}$ and chromium was $54.8 \mathrm{ppb}$. This, combined with the patient's mechanical post-operative symptoms, apparently normal AP and lateral radiographs and the initial suspicion of CSM, prompted further imaging.

Consequently, a low-dose CT scan $(1.4 \mathrm{mSv})$ of the patient's hip was obtained (Fig. 3). The low-dose CT scan was obtained using a SOMATOM Definition AS 128-slice CT scanner (Siemens Heathineers, Erlangen, Germany) using the $\mathrm{CT}$ protocol shown in Table 1. Threedimensional models of the components were produced. The centre of rotation of each component was compared (Fig. 4) and the distance between the edge of the acetabular cup and femoral head surface was measured (Fig. 5). This method works on the premise that a fully engaged head should be equally spaced from the outer surface of the acetabular cup and that the centre of rotation of spheres fitted to the outer surface of the acetabular and femoral head components should overlap, provided that an offset-bore acetabular component has not been used.

Three-dimensional analyses of the CT scan indicated a potential CSM (Figs. 4, 5). Results suggested that the acetabularbearing diameter was $50 \mathrm{~mm}$, highly suggestive of a 2-mm CSM, with the acetabular bearing surface $2 \mathrm{~mm}$ greater than the femoral head. Revision was recommended on this basis.

The company was informed throughout the post-operative follow-up via the local representative. Based on the information that the company had seen they advised against revision surgery and suggested that close clinical follow-up of the patient might be arranged.

The resurfacing was revised 10 weeks post-primary surgery, with the Adept hip resurfacing (MatOrtho, Surrey, UK) exchanged for a Trinity Metafix (Corin, Cirencester, UK). The explanted Adept hip resurfacing components, both acetabular and femoral components, were sent to the London Implant Retrieval Centre for analysis. On visual inspection, implant labelling pointed to a bearing size mismatch (Fig. 6). These measurements were confirmed using a coordinate measuring machine (internal diameter of the cup $=49.98 \mathrm{~mm}$, outer diameter of the head $=47.84 \mathrm{~mm}$ ). Wear analysis of both components was also performed. The volume of wear in the acetabular component was $20.59 \mathrm{~mm}^{3}$. This gives a predicted 1-year wear of $107 \mathrm{~mm}^{3}$. The volume of wear of the femoral head component was $10.14 \mathrm{~mm}^{3}$. This gives a predicted 1-year wear of $47.84 \mathrm{~mm}^{3}$. Under standard walking conditions, with correctly positioned components, assuming 1.9 million cycles per annum [6], the expected wear rate should be less than $2 \mathrm{~mm}^{3} /$ year [7]. 


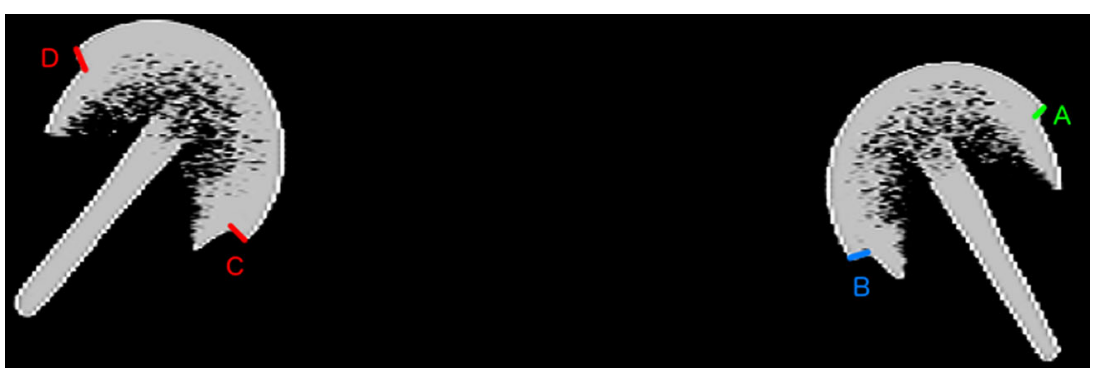

Fig. 3 A 2D CT coronal slice of the patient with the mismatched left hip resurfacing (right) and the patient's previous right hip resurfacing $(l e f t)$ in view. The femoral head of the mismatched component is not sitting

\section{Discussion}

Detection of MoM CSM is very challenging using plain radiographs [2]. However, we have developed a method that uses CT to identify CSM in MoM patients. Our imaging technique includes: 3D CT with metal artefact reduction, a low radiation dose protocol and a software measurement technique [8]. The main limitation of the technique is the requirement to know component specifics regarding dimensions (e.g. if there is a designed offset bore [9]). This poses a problem if the types of component implanted/design specifics of the component are unknown. Another limitation of the technique is that it is time-consuming and requires proficiency in 3D CT analysis software. This may limit its use in clinical practice.

The same principle can be applied to 2D CT slices (Fig. 3), by comparing the distance between the outer surface of the acetabulum and the femoral head (AC-FH). Owing to metal artefact this was only possible at the edge of the acetabulum. For this reason, patients with mismatches due to oversized heads may go undetected because at the acetabular edge oversized femoral heads are centrally located and the AC-FH distance is equal (Fig. 7). We therefore recommend that $3 \mathrm{D}$ models are still generated to accurately visualise the difference between the femoral and acetabular centre of rotations in all planes.

The method was used to detect a mislabelled oversized cup, as the centre of rotation of the acetabular and femoral head components did not overlap, plus the femoral head did not sit

Table 1 Parameters of the low-dose CT protocol

\begin{tabular}{lllll}
\hline Area scanned & $\mathrm{kV}$ & $\mathrm{mAs}$ & Scan length $(\mathrm{cm})^{\mathrm{a}}$ & Collimation \\
\hline ASIS-stem tip & 100 & 100 & 30 & $128 * 0.625$ \\
Knee & 100 & 70 & 20 & $128 * 0.625$ \\
Ankle & 100 & 45 & 10 & $24 * 1.25$ \\
\hline
\end{tabular}

ASIS anterior superior iliac spine

${ }^{a}$ Scan length varies depending upon patient size and component. An anterior-posterior topogram should be acquired to determine scan range. All areas should be scanned using a pitch of 1.0 and rotation time of $1.0 \mathrm{~s}$ and reconstructed using 1-mm slices and the kernel "BONE". An extended scale should be used. centrally in the acetabular component with $B$ being 1.5 times greater than $A$. On the previous right hip resurfacing, with correctly paired components, the femoral head is centrally placed ( $C$ to $D)$

centrally in the acetabular component (Figs. 4, 5). If the femoral component was oversized, the centres of rotation of the acetabulum and femoral head components would not overlap, but the femoral head would remain centrally positioned in the acetabulum (Fig. 7).

Metal ions are known to increase in patients who have MoM implants and there is increasing concern over their toxicity [10]. Furthermore, there is no clear consensus on what counts as normal chromium and cobalt ion levels. The best published evidence has suggested that the normal upper limit of blood metal ions in patients with MoM implants is between 0.5 and $2.5 \mathrm{ppb}$ for chromium and 0.7 and $3.4 \mathrm{ppb}$ for cobalt [11]. Another study indicated serum chromium ion levels above $17 \mathrm{ppb}$ and serum cobalt levels above $19 \mathrm{ppb}$ are likely to result in metallosis [12]. In this case, the patient had metal ions far higher than the normal limit in MoM patients. In addition, the patient would likely have developed metallosis had the detection of CSM and prompt revision not occurred [12].

Currently, proper procedure dictates that only the labels on the boxes are checked and noted. This is because, while the engravings on the acetabulum (Fig. 6) can be seen at revision,

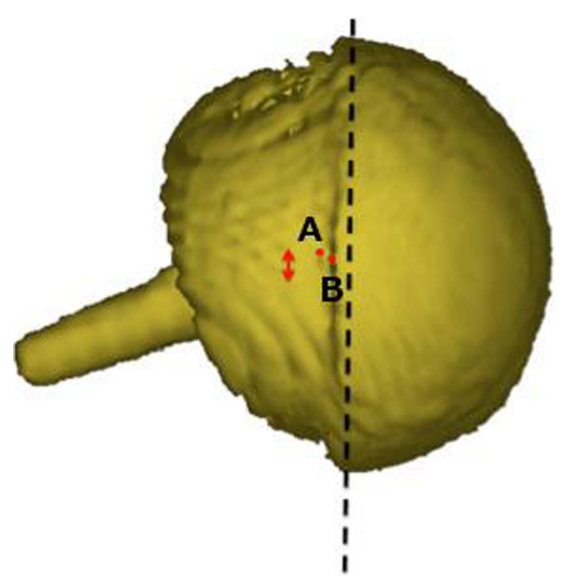

Fig. 4 Three-dimensional model of the patient's metal-on-metal (MoM) resurfacing. The acetabular $(A)$ and femoral head $(B)$ centres of rotation have been marked and the red arrow demonstrates that the two points are not overlapping, which indicated component size mismatch (CSM) 


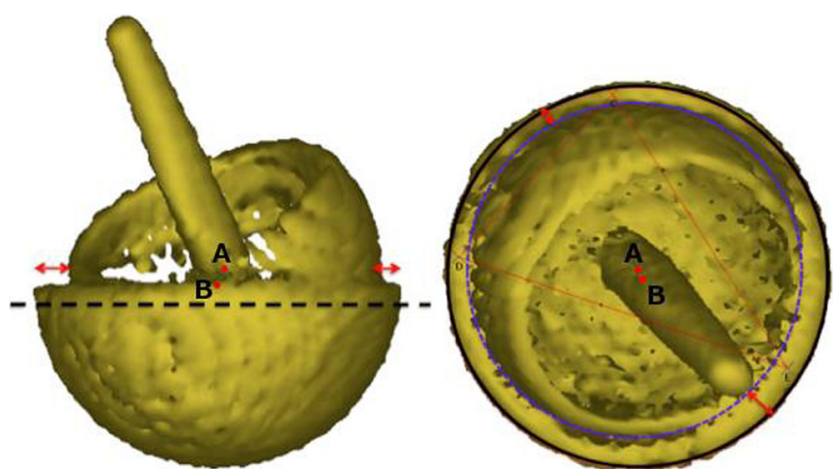

Fig. 5 Lateral (left) and true anterior-posterior of the acetabular cup (right) views of a three-dimensional model of the patient's MoM resurfacing. $A$ is the acetabular centre of rotation and $B$ is the femoral head centre of rotation. The red arrows indicate that the femoral head is not centred in the acetabulum, which suggests CSM

they are not visible before implantation as they are packaged with an impactor cap attached (Fig. 8).

Mislabelling of components has previously been reported on two occasions. In both instances the manufacturer recalled the device, a statement was made by the US Food and Drug Administration and a Medical Device Alert was given by the Medicines and Healthcare Regulatory Agency [13, 14]. Advice from the Medicines and Healthcare Regulatory Agency suggested that the plain radiographs of anyone suspected of being fitted with an incorrect prosthesis should be obtained [14]. However, previous work has demonstrated that CSM may be missed using plain radiographs [2].

Cases of mismatch due to surgical error (mismatching of correctly packaged components) have also previously been reported. However, these cases do not require imaging for detection, provided that the notes are available to be checked.

To reduce the incidence of CSM, we recommend that component sizes are documented on the whiteboard in theatre (in the same way the swab count check is performed). In addition, this case highlights the importance of attempting to check the engravings on the acetabular component after insertion and not just relying on the boxes/stickers. However, this is not

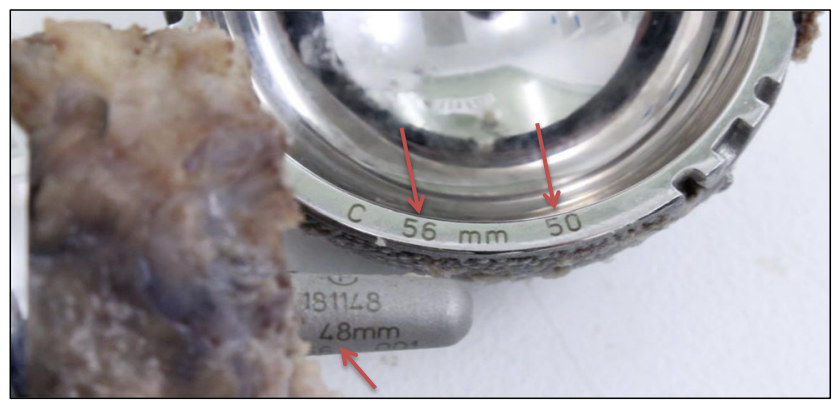

Fig. 6 Photo of the revised Adept hip resurfacing removed from the 53year-old gentleman. Manufacturer labels in the surgical notes record the components as being a 56-mm cup designed for a 48-mm head and a 48$\mathrm{mm}$ head. Engravings (red arrows) and coordinate measuring machine measurements confirm the CSM, as the cup is actually a 56-mm cup designed for a $50-\mathrm{mm}$ head

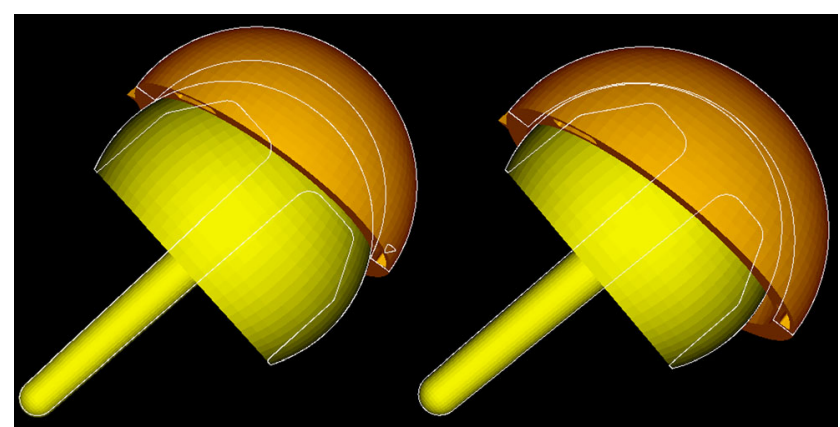

Fig. 7 Two 3D models of potential CSMs: oversized femoral head (left); oversized acetabulum (right). The white outline reveals that the femoral head sits centrally in the acetabulum in cases where the femoral head is oversized. In CSM cases where the acetabulum is oversized (as presented) the femoral head does not sit centrally

always achievable as the implant lies deep in the wound, the engravings are often obscured by blood or overhanging acetabular bone and the engravings may not be on the same side as the surgeon. Furthermore, even if the surgeon saw "56 mm", there are two different acetabular components with an external diameter of $56 \mathrm{~mm}$. The external diameter of $56 \mathrm{~mm}$ is also one of two acceptable pairings that can be used with a $48-\mathrm{mm}$ head. Consequently, both numbers need to be visualised.

Future work will incorporate the lessons learned from this case and retrieval data on mismatches to develop a modified WHO checklist with the aim of reducing the incidence of CSM. Within the modified WHO checklist it would be imperative for the engravings on the components to be directly visualised, either post-implantation or, if this proves too difficult to implement, pre-implantation, following a redesign of the impactor caps.

We applied our method to a patient referred with suspected CSM. Based on our results we recommended revision surgery, and analysis of explanted components confirmed the CSM. To our knowledge, this case is the first report of a CT method being used in a patient to pre-operatively identify CSM. We believe that this CT method will be useful in identifying CSM of MoM components in patients whose surgical notes are unobtainable.

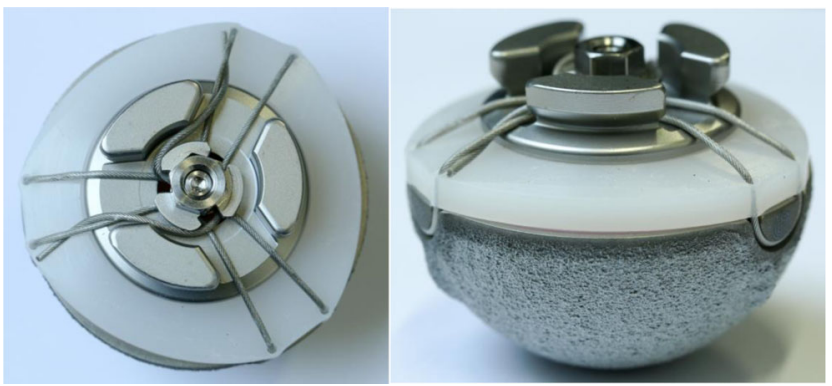

Fig. 8 Photo of an acetabular component with the impactor cap still attached. The impactor cap covers the engravings and is removed only after insertion 
Acknowledgements We would like to thank Robin Richards for the production of Fig. 6. In addition we would like to thank Shiraz Sabah, Harry Hothi and Robert Whittaker for their help in producing this case report.

\section{Compliance with ethical standards}

Ethical approval All procedures performed in studies involving human participants were in accordance with the ethical standards of the institutional and/or national research committee and with the 1964 Declaration of Helsinki and its later amendments or comparable ethical standards.

Informed consent Informed consent was obtained from all individual participants included in the study.

Conflicts of interest The authors declare that they have no conflicts of interest.

Open Access This article is distributed under the terms of the Creative Commons Attribution 4.0 International License (http:// creativecommons.org/licenses/by/4.0/), which permits unrestricted use, distribution, and reproduction in any medium, provided you give appropriate credit to the original author(s) and the source, provide a link to the Creative Commons license, and indicate if changes were made.

\section{References}

1. NHS—never events. Available from: http://www.nrls.npsa.nhs. uk/neverevents/.
2. Whittaker RK, Hexter A, Hothi HS, et al. Component size mismatch of metal on metal hip arthroplasty: an avoidable never event. J Arthroplast. 2014;29:1629-34.

3. Registry NJ. 10th Annual report; 2013.

4. Hanks GA, Foster WC, Cardea JA. Total hip arthroplasty complicated by mismatched implant sizes. Report of two cases. J Arthroplast. 1986;1:279-82.

5. Barrack RL, Burke DW, Cook SD, et al. Complications related to modularity of total hip components. J Bone Joint Surg Br Vol. 1993;75:688-92.

6. Silva M, Shepherd EF, Jackson WO, et al. Average patient walking activity approaches 2 million cycles per year: pedometers underrecord walking activity. J Arthroplast. 2002;17:693-7.

7. Fisher J, Al Hajjar M, Williams S, et al. (v) Simulation and measurement of wear in metal-on-metal bearings in vitro- understanding the reasons for increased wear. Orthop Trauma. 2012;26:253-8.

8. Henckel J, Richards R, Lozhkin K, et al. Very low-dose computed tomography for planning and outcome measurement in knee replacement. The imperial knee protocol. J Bone Joint Surg Br Vol. 2006;88:1513-8.

9. Izquierdo-Avino RJ, Siney PD, Wroblewski BM. Polyethylene wear in the Charnley offset bore acetabular cup. A radiological analysis. J Bone Joint Surg Br Vol. 1996;78:82-4.

10. Keegan GM, Learmonth ID, Case CP. Orthopaedic metals and their potential toxicity in the arthroplasty patient: a review of current knowledge and future strategies. J Bone Joint Surg Br Vol. 2007;89:567-73.

11. De Smet K, De Haan R, Calistri A, et al. Metal ion measurement as a diagnostic tool to identify problems with metal-on-metal hip resurfacing. J Bone Joint Surg Am. 2008;90 (Suppl 4):202-8.

12. Campbell PAOM, Gill HS, DeSmet KA. Interpretation of metal ion levels after metal-on-metal hip resurfacing. Poster P100. New Orleans: American Academy of Orthopaedic Surgeons; 2010.

13. FDA. Product recall notification immediate response required. USA: FDA; 2007.

14. MHRA. Medical device alert. MHRA; 2007. 